\title{
Macro and micro scale modeling of thermal residual stresses in metal matrix composite surface layers by the homogenization method
}

\author{
D. Golanski, K. Terada, N. Kikuchi
}

Abstract The modeling of thermal residual stresses generated in $\mathrm{TaC} /$ stellite and $\mathrm{TiC} /$ stellite composite surface layers produced by the oscillating electron beam remelting on low alloys steel is presented. The homogenization method is applied to analyze the real composite microstructures by utilizing the digital image-based (DIB) geometric modeling technique. Two scales of elastic stress analysis are studied: macroscopic one referring to the global structure of composite layer produced over the substrate of low alloy steel and microscopic, comprising the selected unit cell of composite microstructure.

The results of the analysis show the microscopic stress to be few times higher than the macroscopic one with stress level much above the elastic limit of matrix material, which implies the development of plastic field around the inclusions. The ceramic inclusions within the unit cell are found to be under high compressive stresses. Also, the composite surface layer stays in compression, mainly by the influence of the stress component parallel to the layer/ substrate interface. The effect of hardphase volume fraction is examined and it is found that for a small volume fractions the macro and micro stress does not differ substantially between composites with $\mathrm{TaC}$ and $\mathrm{TiC}$ hardphases despite their mismatch in thermophysical properties. Also, the stress modeling is presented for the composite containing other inclusions and the problem of the selection between 2D and 3D model for the stress analysis is discussed.

1

Introduction

There are several industry fields like aircraft, electrical, nuclear or building where metal matrix composites

Communicated by S. N. Atluri, 14 August 1996

D. Golanski

Visiting Scholar, The Warsaw University of Technology, Welding Department, 85 Narbutta St., 02-524 Warsaw, Poland

K. Terada, N. Kikuchi

The University of Michigan,

Department of Mechanical Engineering,

Computational Mechanics Laboratory, 2350 Hayward,

Ann Arbor, MI 48109-2125, USA

Correspondence to: N. Kikuchi

The authors acknowledge the support of NSF MSS-93-01807, US Army TACOM, DAAE07-93-C-R125 and US Navy ONR, N00014-94-1-0022 and AFOSR-URI program, DoD-G-F49620-930289 and DEKABAN fund at The University of Michigan.
(MMC) are preferably used for engineering applications reducing the use of traditional materials. High strength of MMC together with high stiffness and high thermal stability at elevated temperatures makes them very attractive for engineering structures.

One of the fields where application of MMC can be beneficial are composite surface layers produced over a substrate of conventional material. The role of a surface layer is to secure the surface of material against the action of external factors like corrosion as well as mechanical factors (intensive wear, cycling loading) resulting in the excessive wear of material during service life (Rohatgi et al. (1994)). An example of application of composite surface layers is for many machine parts and tools (e.g. drilling and boring tools) which are often subject to extreme working conditions. Although the commonly used materials for such applications are characterized by high strength, wear and corrosion resistance, yet there is still need to improve the service condition for this kind of layers because of the increase of working temperatures and loads. The metal matrix composite may be suitable for this purpose as their unique properties can sustain these demanding service conditions. By embedding hard ceramics in the form of fibers, whiskers or particulates into the metal matrix, we can expect the hard inclusions to carry on the main load resulting from the intensive surface wear during the service life of machine parts. In such a system, elastic ceramic hardphases will allow to absorb the dynamic load much better than the metal matrix, which can be damaged through the accumulation of loads (see e.g., Broutman and Krock (1974)).

The proper selection of materials for a composite layer over a given substrate material is very important as this process should take into account many factors, of which the most important is chemical, metallurgical and physical compatibility between composite layer and the substrate material and also between the composite components themselves. To attain metallurgical compatibility we may compose a composite with matrix material taken as the metal alloy traditionally used for such layers. In this meaning the surface material is enriched with hard inclusions creating the composite with unique properties.

On the other hand, chemical compatibility requires the matrix and reinforcement to be at the equilibrium state in thermodynamically stable phases. This can be achieved by the proper selection of the reinforcing phase for a given metal matrix. Usually ceramic materials like $\mathrm{SiC}, \mathrm{TiC}, \mathrm{TiB}_{2}$, $\mathrm{ZrO}_{2}, \mathrm{Al}_{2} \mathrm{O}_{3}$ are used as a hardphase while the metal matrix may be selected from titanium, aluminium, magnesium or nickel alloys. Such combination of constituents 
will not assure the physical compatibility between composite components and also between the composite layer and the substrate material. The most important is the problem of mismatch in thermal expansion coefficients between the reinforcement and matrix components. When a ductile matrix has higher thermal expansion coefficient than the reinforcement, then during formation of the composite the hard phase contains compressive residual stresses which seems to be preferable for brittle ceramic materials although the subsequent external loads may reverse the sign of these stresses. For the matrix materials with high yield stress it is advised to select reinforcing materials with thermal expansion coefficient close to the matrix, because the limited plasticity can lead to high internal stresses. However, the presence of residual stresses in composites can be beneficial or detrimental depending upon the selection of constituent materials for the matrix and hardphase. An example of positive effect of residual stresses is aluminum matrix composite reinforced with $\mathrm{SiC}$ particles. In this system, residual stresses induce the formation and further movement of dislocation in the plastic aluminum matrix, which leads to the strengthening of $\mathrm{Al}$ and subsequent residual stress relaxation (Shi and Arsenault (1994)). The negative effect can be seen for composites with decreased matrix plasticity and brittle reinforcements. In this case the composite can be damaged by the formation and growth of microcracks both through the matrix and the reinforcement. It was observed by MacKay (1990) for TiAl composites reinforced with $\mathrm{SiC}$ particles ${ }^{4}$.

The stress state is one of the key factors affecting the reliability and durability of the composite structure. We can distinguish two-scale levels of stress in the system of composite surface layers. The first one refers to the microscopic level of stress state within the composite and the other refers to the global structure, where the composite layer is treated as a homogeneous body with uniform properties. Both scales of stress are important parts of a stress analysis in this system. The knowledge of stress state can be especially desired in the process of controlling the properties of manufactured composites (composite layers) and their thermomechanical response under applied loads.

However, the investigation of stress state in composites is difficult because of the existence of different phases in one material. Thus analytical and numerical methods grew up to determine the stresses. There are several, less or more precise, analytical models used to predict the overall composite thermomechanical response. These can include "the rule of mixture", "shear lag model" or "Eshelby's equivalent inclusion model"(see e.g., Taya and Arsenault (1989)). The numerical methods are primary based on the finite element method. The finite element model usually represents a small scale region with matrix and reinforcement repeatedly distributed in a whole structure and the computed stresses are related to the microscopic stage of the problem. When a composite has randomly distributed reinforcements, which is usually the case for the particulate reinforced composites, the problem of determining micro and macroscopic stress is more complicated with the mentioned methods. To estimate the stress state in real randomly distributed particles in the matrix more sophisticated methods have been utilized. One of the promising and advanced ones is the homogenization method as it allows to describe both the micro and macro thermomechanical behavior of composite materials using rigorous mathematical theory (Guedes and Kikuchi (1990) and Cheng (1992)). It should be noted that the theory can describe both micro- (local) boundary value problems with the help of the two-scale asymptotic expansion method if the periodic boundary condition is introduced on the representative volume element (RVE) (see SanchezPalencia (1980)). Therefore, we assume that the selected composite layers are statistically homogeneous and periodic so that the asymptotic homogenization method can be applied if the RVE size is sufficiently large.

On the other hand, the continuum-based formulation of the homogenization method requires some kind of numerical methods such as finite element method. To carry out the computation effectively, modeling of appropriate microstructural geometry is essential since most of metal matrix composites have more or less random nature in their microstructural morphology. In this context, Hollister and Kikuchi (1994) addressed the modeling issue in FE geometric modeling of bone microstructure. They extensively utilized the digital images and their processing techniques to construct the digitized 3D-FE model of a bone microstructure by identifying each voxel as an finite element. Along with the asymptotic homogenization method, their digital image-based (DIB) modeling technique enabled the quantitative study of the macro- and micromechanical characteristics of bone's porous skeleton in the framework of linear elasticity. In order to successfully take into account the effect of microstructural geometry, we shall utilize this novel method in our homogenization analyses. This method has been used in our work and the fromulae in linear elasticity will be presented later in the paper.

The aim of this paper is to present some aspects on modeling the stress state in metal matrix composite surface layers through the utilization of DIB geometric modeling. This technique allows to analyze the real composite microstructures and to convert them into virtual representation of 3D geometry as shown by Terada et al. (1996). For the stress analysis, we have selected nickel-base alloy as the matrix and four carbide ceramic materials: $\mathrm{SiC}$, $\mathrm{TiC}, \mathrm{TaC}$ and $\mathrm{HfC}$ for the hardphase. The homogenization method is used to evaluate the effective material properties such as elastic and thermal expansion coefficient matrices by taking a "unit cell" as a representative volume element. Also, other simplified models used to estimate the overall composite properties are presented for comparison. We have conducted the analysis for linear elasticity case with thermal loading equal to the temperature difference between composite formation and room temperature, therefore we analyzed the thermal residual stresses generated upon cooling of composite layer. For high temperature drops, the thermal residual stresses may significantly influence the overall stress state in the composite layer as well as in the whole structure as they shall superimpose with the external service loads. The application of the homogenization method allows us to estimate the stress state in the composite's unit cell at the micro- 
scopic level by localizing the deformation of the global structure, which has been obtained in the stress analysis.

2

\section{Materials and processing}

For the purpose of the analysis we based our work on the composite with stellite (nickel-based alloy) as a matrix and carbide ceramics as inclusions. Stellites are widely used for surface layers produced by plasma hardfacing method since they have high wear and corrosion resistance. The chemical composition of the stellite used in the analysis is equivalent to Deloro ${ }^{\circledR}$ Stellite 40 . The following properties of the stellite were assumed in the analysis: density $\rho=8330 \mathrm{~kg} / \mathrm{m}^{3}$,Young's modulus $\mathrm{E}=183 \mathrm{GPa}$, Poisson's ratio $v=0.3$ and coefficient of thermal expansion $\alpha=9.7 \cdot 10^{-6} 1 / \mathrm{K}$ according to technical data of Deloro ${ }^{\circledR}$ Stellite (1970).

The selection of reinforcing phase depends on many factors affecting the properties of metal matrix composites. We have assumed four carbide particles: $\mathrm{TaC}, \mathrm{TiC}, \mathrm{SiC}$ and $\mathrm{HfC}$ as the reinforcement. Such selection was dictated by the fact that the contribution of carbide metallic bonds should effectively improve the wetting of ceramic particles by the stellite matrix. Also, because the composite surface layers were obtained in the process of electron beam remelting the high formation entalphy of these ceramics prevents their decomposition during remelting.

The properties of ceramics were taken from the literature (ASM Reference Book and Morrell (1989)) and are presented in Table 1. As it is seen from the table the selected ceramics differ in their properties. Specifically, HfC and $\mathrm{TaC}$ have high density which is important when we take into account the stiffness/density ratio as a common indicator of composite weight with a given stiffness. The thermal expansion coefficient $(\alpha)$ of the hardphases, which has the strong influence on the stress state generated within a composite varies between ceramics in a wide range from 4 to $7.4 \cdot 10^{-6} 1 / \mathrm{K}$, while the Young's modulus has similar values for all except TaC. All considered ceramics have high melting temperature and high hardness which makes them attractive for applications working at elevated temperatures.

One of the advanced methods of producing this kind of composite layers is a technique of remelting by a high energy concentrated beam (electron, laser or plasma) the surface of substrate material previously covered with a mixture of metal and ceramic powders. The process of

Table 1. The properties of ceramic reinforcements used for the metal matrix composites

\begin{tabular}{|c|c|c|c|c|}
\hline \multirow[t]{2}{*}{ Properties } & \multicolumn{4}{|c|}{ Ceramics } \\
\hline & $\mathrm{SiC}$ & $\mathrm{HfC}$ & $\mathrm{TaC}$ & $\mathrm{TiC}$ \\
\hline Melting temperature (K) & 2500 & 4203 & 4152 & 3340 \\
\hline Density $\rho\left(\mathrm{g} / \mathrm{cm}^{3}\right)$ & 3.10 & 12.67 & 14.50 & 4.92 \\
\hline $\begin{array}{l}\text { Coefficient of thermal } \\
\text { expansion } \alpha\left(10^{-6} 1 / \mathrm{K}\right)\end{array}$ & 4.02 & 6.60 & 6.3 & 7.4 \\
\hline Young's modulus $E$ (Gpa) & 402 & 470 & 722 & 447 \\
\hline Poisson's ratio $v$ & 0.142 & 0.180 & 0.240 & 0.190 \\
\hline Shear modulus $G$ (Gpa) & 178 & 193 & 227 & 186 \\
\hline Bend strength (Mpa) & 459 & $234-241$ & $215-310$ & $282-667$ \\
\hline Microhardness (Gpa) & 28 & $22.6-30.5$ & $16-24$ & $20-24$ \\
\hline
\end{tabular}

coverage the surface with the powders can be done manually, by spreading the powders over the surface or mechanically using e.g. the plasma spraying method (see e.g., Matejka (1989)). In fact, the former technique may be used separately for a direct deposition of composite layers, but because of the layer porosity and adhesive bonding to the substrate it still needs a subsequent remelting in order to reduce the porosity and create strong bonds at the layer/ substrate interface.

In our analysis we have utilized the microstructures of carbide ceramics/stellite composites produced by the oscillating electron beam remelting technique over the substrate of low alloy steel (equivalent to ASTM A618 grade). Particularly, we based our work on $\mathrm{TaC} /$ stellite and $\mathrm{TiC} /$ stellite composites as the initial source for the further analysis. The microstructures of these composite layers are presented in Fig. 1. According to the DIB geometrical modeling described in the next section we have specified the composite's unit cell by selecting them from the images of composite micrographs, which is marked and shown in Fig. 1.

\section{3}

The homogenization method and microstructural modeling by digital images

\section{1}

Introduction

In this section, we shall present two rigorous tools to analyze the mechanical behavior of the MMC whose material properties and configurations are described in the previous section. One of the tools is the mathematical theory of homogenization and the other is the Digital Image-Based (DIB) geometric modeling technique.

The so-called asymptotic homogenization method concerns composite media whose microstructure occupies a fixed region with characteristic length $\varepsilon$ of its heterogeneity. The theory asserts that if the selected representative volume element (RVE) is infinitesimally small, the actual displacement, $\boldsymbol{u}^{\varepsilon}$, tends to the homogenized displacement field, $\boldsymbol{u}^{0}$, which is the global solution of the governing equations whose coefficients have been homogenized. While the effective properties can be derived from the micromechanical characteristics, the micromechanical behaviors can be obtained by localizing the overall structural response to the local one; these processes are called, respectively, homogenization and localization. The global-local approach was successfully applied to the engineering problems in both linear elasticity and elastoplasticity with the help of Finite Element Method (Guedes and Kikuchi (1990), Devries and Léne (1987), Duvaut and Nuc (1983)).

After deriving the homogenization formulae for linear elasticity with temperature change, the procedure of construction of a unit cell by the DIB modeling is briefly described.

\section{2}

The homogenization formulae

We recall that the theory concerns statistically homogeneous or periodic composite media of domain $\boldsymbol{\Omega}^{\varepsilon}$ and 


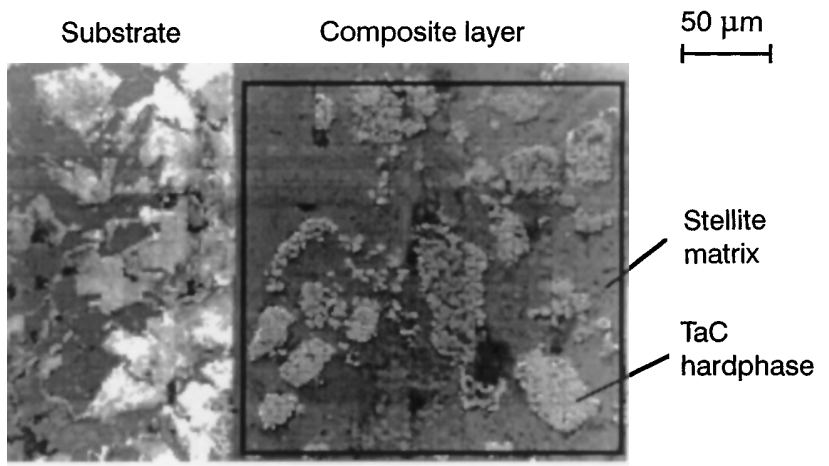

Low alloy steel

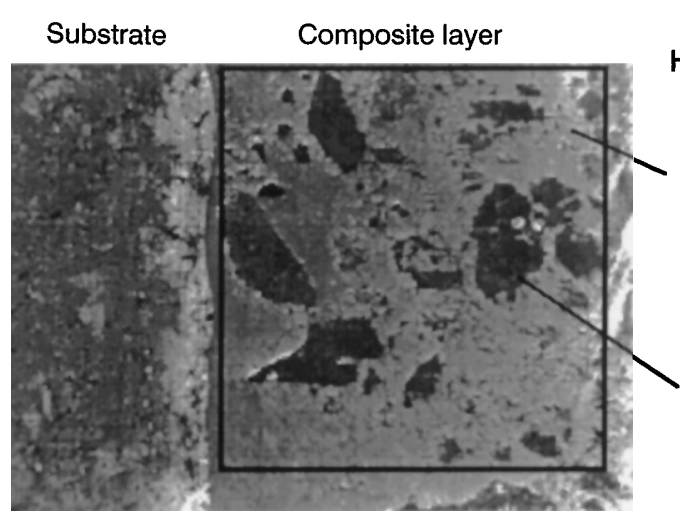

Low alloy steel

b

Fig. 1a,b. The microstructure of $\mathrm{TaC} /$ stellite (a) and $\mathrm{TiC} /$ stellite (b) composite surface layers produced on low alloy steel by the electron beam remelting method

the representative volume element (RVE) occupying a microscopic region $\mathrm{V}$ with characteristic length $\varepsilon$. Identifying the size of the RVE with $\varepsilon$, we introduce two different scales: one of these is a macroscopic scale denoted by $x$, in the domain $\boldsymbol{\Omega}^{\varepsilon}$ at which the heterogeneities are invisible and the other one is an microscopic one denoted by $y=x / \varepsilon$ which enlarges the RVE region by $\varepsilon$ such that $\mathrm{V}=\varepsilon \mathrm{Y}$. Thus, the superscripts introduced in variables indicate their orders as well as the dependency on both $x$ and/or $y$. Let the structure be subjected to a surface traction $\hat{t}$ and a prescribed homogenous displacement boundary conditions on $\Gamma_{t}$ and $\Gamma_{u}$, respectively, with temperature change $\Delta T$. According to the principle of minimum total potential energy for equilibrium, the displacement $\boldsymbol{u}^{\varepsilon}$ is the solution of the variational problem defined in the domain $\boldsymbol{\Omega}^{\varepsilon}$ :

$$
\begin{aligned}
\int_{\Omega^{\varepsilon}} \boldsymbol{\varepsilon} & \left(\boldsymbol{v}^{\varepsilon}\right): \boldsymbol{D}^{\varepsilon}(\boldsymbol{x}): \boldsymbol{\varepsilon}\left(\boldsymbol{u}^{\varepsilon}\right) \mathrm{d} x \\
= & \int_{\Omega^{\varepsilon}}\left(\Delta T \boldsymbol{\varepsilon}\left(\boldsymbol{v}^{\varepsilon}\right): \boldsymbol{D}^{\varepsilon}: \boldsymbol{\alpha}^{\varepsilon}+\boldsymbol{b}^{\varepsilon} \cdot \boldsymbol{v}^{\varepsilon}\right) \mathrm{d} x^{\varepsilon} \mathrm{d} x, \forall \boldsymbol{v}^{\varepsilon} \\
& +\int_{\Gamma_{t}} \hat{\boldsymbol{t}}(x) \cdot v
\end{aligned}
$$

with the constitutive relation

$$
\begin{aligned}
\boldsymbol{\sigma}^{\varepsilon} & =D^{\varepsilon}(\boldsymbol{x}):\left(\boldsymbol{\varepsilon}\left(\boldsymbol{u}^{\varepsilon}\right)-\Delta T \boldsymbol{\alpha}^{\varepsilon}(\boldsymbol{x})\right) \\
& =\boldsymbol{D}^{\varepsilon}(\boldsymbol{x}): \boldsymbol{\varepsilon}\left(\boldsymbol{u}^{\varepsilon}\right)-\Delta T \boldsymbol{\beta}^{\varepsilon}(\boldsymbol{x})
\end{aligned}
$$

Here $\boldsymbol{v}^{\varepsilon}$ is the virtual displacement, $\boldsymbol{b}^{\varepsilon}(\boldsymbol{x})$ the body force, $D^{\varepsilon}(x)$ the elasticity tensor, $\alpha^{\varepsilon}(x)$ the coefficient of thermal expansion (CTE) with $\boldsymbol{\beta}^{\varepsilon}=\mathbf{D}^{\varepsilon}: \boldsymbol{\alpha}^{\varepsilon}$.

With the help of the method of two-scale asymptotic expansion (see, e.g., Sanchez-Palencia (1980)), the theory asserts that if the selected RVE is periodic and infinitesimally small, the actual displacement, $\boldsymbol{u}^{\varepsilon}$, tends to the homogenized one, $\boldsymbol{u}^{\boldsymbol{0}}$, which is the solution of the following macroscopic equations whose coefficients have been homogenized.

$$
\begin{aligned}
\int_{\Omega} \varepsilon_{x}(\boldsymbol{v}): \mathrm{D}^{H}: \varepsilon_{x}\left(\boldsymbol{u}^{0}\right) \mathrm{d} x= & \int_{\Omega} \Delta \boldsymbol{T} \varepsilon_{x}(\boldsymbol{v}): \mathrm{D}^{H}: \alpha^{H} \mathrm{~d} x \\
& +\int_{\Omega} \boldsymbol{b}^{H} \cdot \boldsymbol{v} \mathrm{d} x+\int_{\Gamma_{t}} t \cdot v d \Gamma \quad \forall \boldsymbol{v}
\end{aligned}
$$

and that the Y-periodic characteristic deformations $\chi^{k h}$ and $\psi$ can be obtained by solving the following microscopic equations, respectively:

$$
\begin{aligned}
& \int_{Y} \boldsymbol{\varepsilon}_{y}(\boldsymbol{w}): \boldsymbol{D}: \boldsymbol{\varepsilon}_{y}\left(\chi^{k h}\right) \mathrm{d} y \\
& =\int_{Y} \boldsymbol{D}: \boldsymbol{\varepsilon}_{y}(\boldsymbol{w}) \mathrm{d} y, \forall \boldsymbol{w} ; \mathrm{Y}-\text { periodic } \\
& \int_{Y} \boldsymbol{\varepsilon}_{y}(\boldsymbol{w}): \boldsymbol{D}: \boldsymbol{\varepsilon}_{y}(\boldsymbol{\psi}) \mathrm{d} y \\
& \quad=\int_{Y} \boldsymbol{\beta}: \boldsymbol{\varepsilon}_{y}(\boldsymbol{w}) \mathrm{d} y, \forall \boldsymbol{w} ; \mathrm{Y}-\text { periodic }
\end{aligned}
$$

Here, the strains are denoted by $\boldsymbol{\varepsilon}_{\gamma}=1 / 2\left(\boldsymbol{v} \nabla_{\gamma}+\nabla_{\gamma} \boldsymbol{v}\right)$ $(\gamma=x$ or $y)$ and the homogenized quantities in Eqn. (3) are calculated by averaging over the unit cell:

$$
\begin{aligned}
\boldsymbol{D}^{H}= & \frac{1}{|Y|} \int_{Y} \boldsymbol{D}: \boldsymbol{s} \mathrm{d} y, \boldsymbol{\beta}^{H}=\frac{1}{|Y|} \int_{y}\left(\boldsymbol{\beta}-\boldsymbol{D}: \boldsymbol{\varepsilon}_{y}(\boldsymbol{\psi})\right) \mathrm{d} y \\
& \text { and } \boldsymbol{b}^{H}=\frac{1}{|Y|} \int_{Y} \boldsymbol{b} \mathrm{d} y
\end{aligned}
$$

Also, the tensor of localization have been defined as $s_{i j}^{k h}=I_{i j}^{k h}-\varepsilon_{y, i j}\left(\chi^{k h}\right)$ where $I_{i j}^{k h}$ indicates the fourth order identity tensor. Once the macroscopic displacement $\boldsymbol{u}^{o}$ and $\Delta T$ are obtained in the macroscopic region, these values are localized to give the micromechanical response of the unit cell. Therefore, the microscopic stress is defined by

$\boldsymbol{\sigma}^{o}(\boldsymbol{y})=[\boldsymbol{D}(\boldsymbol{y}): \boldsymbol{s}(\boldsymbol{y})]: \boldsymbol{\varepsilon}_{x}\left(\boldsymbol{u}^{o}\right)-\Delta T\left(\boldsymbol{\beta}(\boldsymbol{y})-\boldsymbol{\varepsilon}_{y}(\boldsymbol{\psi})\right.$

On the other hand, the macroscopic quantities are obtained by taking the average over the RVE domain Y. In order that the macroscopic strain $\boldsymbol{\varepsilon}_{x}\left(\boldsymbol{u}^{o}\right)$ would be the volume average of the microscopic one $\boldsymbol{e}^{o}$, it seems ap- 
propriate that the RVE domain and the microscopic displacement $\boldsymbol{w}^{1}$ would be periodic and therefore so are $\chi^{k h}$ and $\psi$. In this way, we may call the RVE the unit cell.

If we postulate that the displacement were Y-periodic, the substitution of the variables obtained in the above provides the following microscopic governing equations:

$$
\begin{aligned}
\int_{Y} \boldsymbol{w} & \nabla_{y}: \mathbf{D}: \nabla_{y} \chi^{k h} \mathrm{~d} y \\
& =\int_{Y} \mathbf{D}: \nabla_{y} \boldsymbol{w} \mathrm{d} y, \forall \boldsymbol{w}: \mathrm{Y}-\text { periodic }
\end{aligned}
$$

and

$\int_{Y} \boldsymbol{w} \nabla_{y}: \mathbf{D}: \nabla_{y} \psi \mathrm{d} y=\int_{Y} \boldsymbol{\beta}: \nabla_{y} \boldsymbol{w} \mathrm{d} y$

Using these solutions $\chi^{k h}$ and $\psi$, the homogenized elasticity tensor and the homogenized CTE are constructed by the following formulae:

$\mathbf{D}^{H}=\frac{1}{|Y|} \int_{Y} \mathbf{D}: s \mathrm{~d} y$

$\boldsymbol{\alpha}^{H}=\left(\mathbf{D}^{H}\right)^{-1}:\left[\frac{1}{|Y|} \int_{Y}\left(\boldsymbol{\beta}-\mathbf{D}: \boldsymbol{\varepsilon}_{y}(\boldsymbol{\psi})\right) \mathrm{d} y\right]$,

and

$\boldsymbol{b}^{H}=\frac{1}{|Y|} \int_{Y} \boldsymbol{b} \mathrm{d} y$.

where $|Y|$ indicates the volume of the unit cell.

Thus, the effective properties can be derived from the micromechanical characteristics and the micromechanical behaviors can be obtained by localizing the overall structural response to the local one; these processes are called the homogenization and the localization, respectively.

We have skipped several steps in the formulation and omitted some explanations, since they are not relevant to our present interest. One can refer to literature, for example, by Guedes and Kikuchi (1990) Devries and Léne (1987) or Duvaut and Nuc (1983) for the detailed derivation of the homogenization formulae.

\section{3}

Digital image-based FE-geometric modeling for the unit cell Digital image-based (DIB) technique is used to catch and manipulate the image of composite microstructures (unit cells) so that they could be analyzed by the homogenization method. Also, this technique is very helpful for preparing the images of composite unit cells to some other additional processing like generation of $3 \mathrm{D}$ structures or changing volume fraction of inclusions. The main procedure of preparation the unit cell of a composite microstructure can be divided into the following major four parts (see Fig. 2):

1. Capturing and Sampling: prior to digitalization, an image of a composite microstructure must be captured by an optical sensor which is chosen upon the desired formation modality. This process is assumed to be done by a high resolution scanner unit.

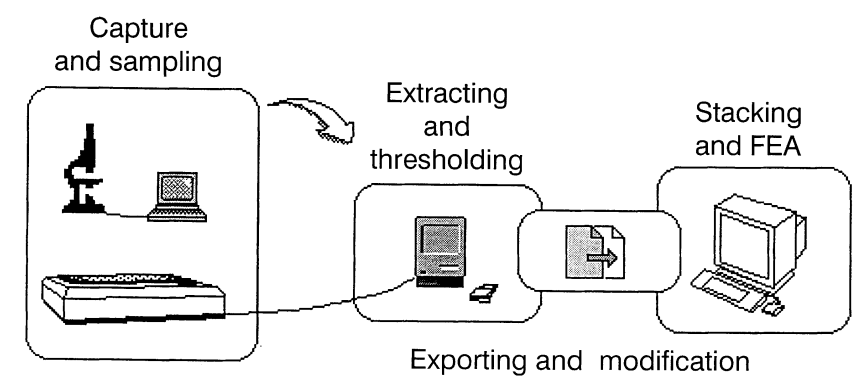

Fig. 2. The diagram showing DIB modeling sequence

2. Selecting and Thresholding: which are probably the most important operations. This process determines the unit cell size, its FE model size and the microstructural configurations. By giving the thresholding pixel value, the actual morphology such as inclusion shape, volume fractions, etc., is determined.

3. Exporting (and Adjusting, if necessary): the binary data stored in the computer are exported into an ASCII file and transferred to a UNIX platform so that our computer program can read and recognize the data. This set of data is actually the prototype of our FE model, which is either two or three dimensional. Depending on our needs, the microstructural configuration is adjusted, e.g., the volume fraction is modified by changing the number of pixels.

4. Stacking: prior to or during the finite element analysis (FEA), the process is made using the exported data to construct the three-dimensional (3D) structure.

While the first three stages correspond to the pre-processing of FEA, the last process includes both the main part and post-processing of the FEA of the homogenization method. In order to construct 3D FE model in the DIB modeling, two dimensional digital images have to be combined. The forth process, namely the stacking, corresponds to such data operation. In 3D FE modeling, each pixel in a 2D image is recognized as a voxel which is identified as a finite element in FEA. Then, the FE model obtained in this process is the direct interpretation of the scanned image using two dimensionally presented micrographs of real composite materials along with image-processing software. Therefore, the homogenization analyses can reflect the effects of the original geometric configuration.

This method involves image processing which fully utilizes both hardware and software capability available. For our particular purpose, the process of changing the volume fraction of a constituent can be easily done by operating the voxel values of the digitized unit cell model. The more detailed description of the DIB modeling procedure, the related image processing and some applications to the homogenization analyses are found in Terada et al. (1996).

\section{4}

The procedure of calculations and analysis

\section{1}

Calculation scheme

We have implemented the following scheme for calculation of macro and microscopic residual stresses in the 


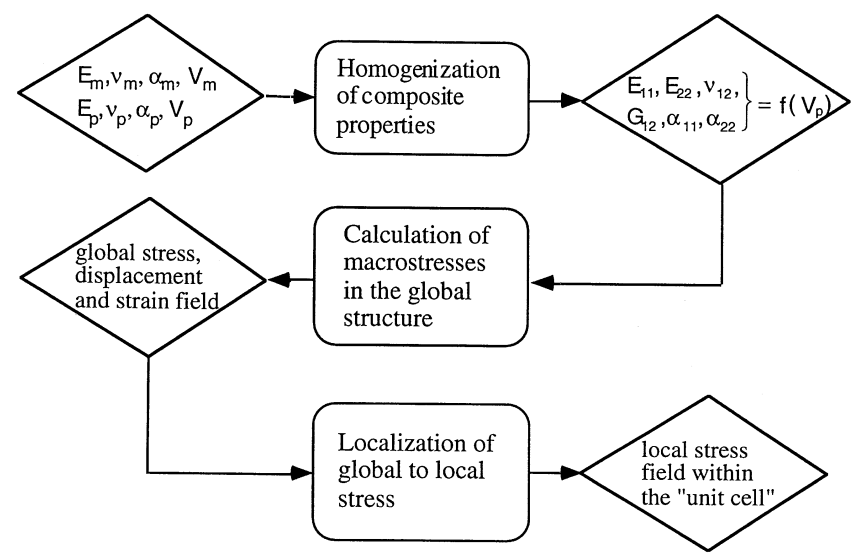

Fig. 3. The scheme of calculation procedure applied in the stress analysis

MMC surface layers shown in Fig. 3. First, the homogenization method is used to obtain the effective composite properties dependent on the properties of its constituents and their volume fraction. This is done for the selected unit cell taken from the microstructure of composite. Next, the global structure is defined to compose the substrate material covered with a composite surface layer having uniform homogenized properties. Then, the standard finite element method is applied to solve for the displacement and stress field in the macroscopic scale (global structure). The last step utilizes calculated global displacement field taken for the selected unit cell from the composite's global structure to computer the microscale (local) stress distribution for composite microstructure represented by the selected unit cell.

\section{2}

\section{Changing volume fraction}

The initial hardphase volume fraction of $\mathrm{TaC} /$ stellite and $\mathrm{TiC} /$ stellite equaled approximately $30 \%$ based on the weight method. In the process of digital image-based (DIB) modeling we have selected the unit cell as a representative volume element from $\mathrm{TaC} /$ stellite and $\mathrm{TiC} /$ stellite composites (Fig. 1). After thresholding and adjusting we have obtained digitized image of the selected composites' microstructures as seen in Fig. 4. These images contain 30450 pixels for TaC/stellite and 29225 pixels for TiC/stellite composites. The calculated volume fractions for these unit cells equaled 21 and $24 \%$, respectively.

To see the effect of volume fraction on the thermomechanical response of selected composites we have generated other volume fractions upon those digitized unit cells. As the typical hardphase volume fraction in MMC ranges from 5 to $40 \%$ we have generated four more unit cells with volume fractions of $5,13,30$ and $40 \%$ covering the range of application. Fig. 5 shows the unit cells with 5\% and $40 \%$ hardphase volume fraction for $\mathrm{TaC} /$ stellite composite as an example. The procedure we applied for the volume fraction change randomly removes/adds hardphase material (pixels) from/to the inclusion-matrix boundary. As it is seen from these pictures, rather torn shape boundary of inclusions is generated, but for the purpose of the analysis this would not be a significant factor.

\section{5}

\section{Results of calculations}

\section{1}

\section{Effective composite properties}

By applying the homogenization method we have calculated the engineering composite's constants for the plane stress case: Young's modulus $\left(E_{11}, E_{22}\right)$ Poisson's ratio $\left(v_{12}\right)$, shear modulus $\left(G_{12}\right)$ and thermal expansion coefficient $\left(\alpha_{11}, \alpha_{22}\right)$. There are many models found in the literature elaborated to estimate the effective properties of composites (see e.g., Vaidya and Chawla (1994) or Whitney and McCullough (1990)). For comparison with the homogenization method we have included the calculated effective composite properties based on the classical "rule of mixture" used primarily for the transverse direction of fiber composites and two other often used models based on approximated equations for particulate composites.

These models are presented as follows:

For the estimation of Young's modulus we used "the rule of mixture" model by Reuss (1929):

$E_{c}=\frac{1}{V_{m} / E_{m}+V_{p} / E_{p}}$
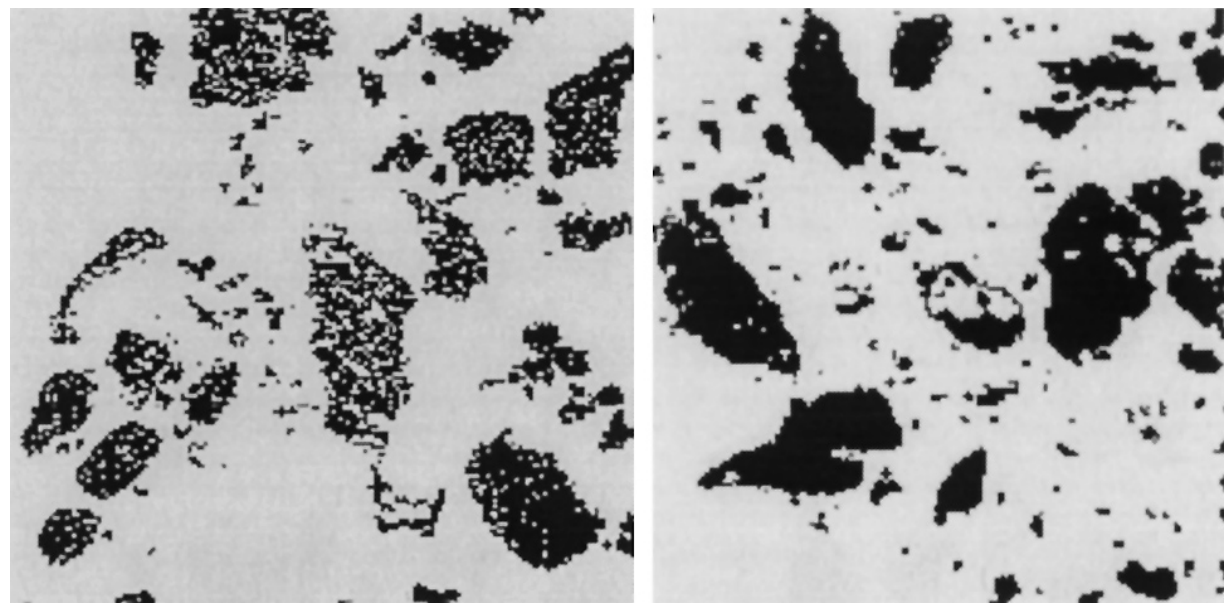

Fig. 4. The digitized unit cells of $21 \%$ $\mathrm{TaC} /$ stellite (left) and 24\% TiC/stellite composites (right) after selection and thresholding 

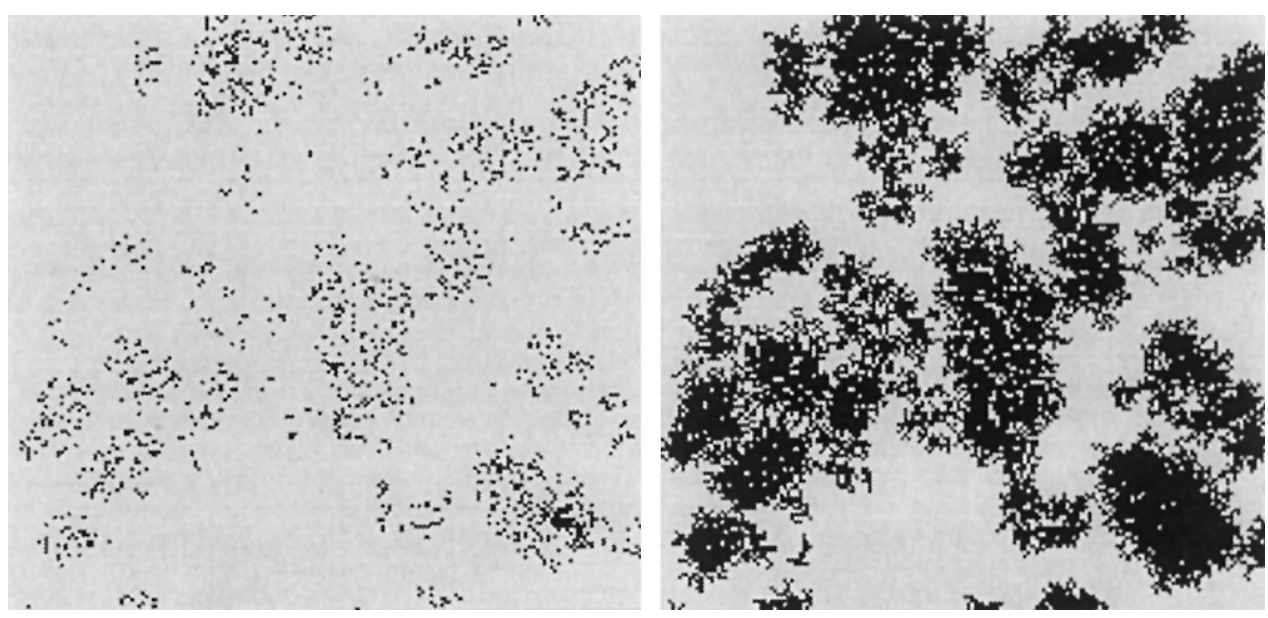

Fig. 5. TaC/stellite digital image with generated hardphase volume fraction of $5 \%$ (left) and $40 \%$ (right) and Halpin-Tsai (Halpin and Kardos (1967)) expression:

$E_{c}=\frac{E_{m}\left(1+\xi \eta V_{p}\right)}{1-\eta V_{p}}$ where $\eta=\frac{\left(E_{p} / E_{m}-1\right)}{\left(E_{p} / E_{m}+\xi\right)}$

where: $E$ - Young's modulus, $V$ - volume fraction $(c-$ composite, $m$ - matrix, $p$ - particulate), $\xi$ is factor assumed to be 1 .

For the estimation of thermal expansion coefficient we used two models:

Kerner's (1956) model for volumetric coefficient of thermal expansion $\beta=3 \alpha$ :

$$
\begin{aligned}
\beta_{c}= & \beta_{m} V_{m}+\beta_{p} V_{p}-\left(\beta_{m}-\beta_{p}\right) V_{m} V_{p} \\
& \times \frac{1 / K_{m}-1 / K_{p}}{V_{m} / K_{p}+V_{p} / K_{m}+3 G_{m} / 4}
\end{aligned}
$$

where $K$ is bulk modulus and $G$ shear modulus.

This model accounts for shear and isostatic stresses, but the last part of this expression can be particularly negligible reducing the formula for $\alpha$ to the classical "rule of mixture":

$\alpha_{c}=\alpha_{m} V_{m}+\alpha_{p} V_{p}$

Turner's model (1946), which accounts for the case of hydrostatic stresses:

$\alpha_{c}=\frac{\alpha_{m} V_{m} K_{m}+\alpha_{p} V_{p} K_{p}}{V_{m} K_{m}+V_{p} K_{p}}$

The calculated homogenized composite properties were combined and plotted in Fig. 6 with the presented simplified models. These models are used for comparison purposes only. The measurement results are necessary to validate the analytical ones, although good agreement is obtained for Halpin-Tsai and Turner models. It can also be seen from the figure that the effective properties both of $E$ and $\alpha$ are close each other for a small volume fraction. The increase of the hardphase volume fraction results in bilinear increase of the homogenized properties and the higher influence of ceramic properties on the effective properties of the composite which is seen especially for the $\mathrm{TaC}$ ceramic having high Young's modulus.
The "rule of mixtures" model gives a very rough estimation of composite properties except for the case of effective density of composite which gives accurate approximation. The density of a composite plays an important role when we are taking into account stiffness/ density (specific stiffness) ratio. Most ceramics are characterized by a higher Young's modulus and lower density than matrix material. This feature is used to produce composites structures that are lighter than traditional materials or have reduced cross section without the loss of stiffness. Chawla (1987) discussed a simple model which shows that for beams under compressive and flexural loads the minimum weight of composite structure for a given stiffness can be obtained while the term $\left(E / \rho^{2}\right)$ reaches maximum.

Fig. 7 shows the change of specific stiffness of $\mathrm{TaC} /$ stellite and TiC/stellite composites. The Young's modulus was obtained by the homogenization method while the density of composite was estimated by the "rule of mixture":

$\rho_{h}=\rho_{p} \cdot V_{p}+\rho_{m} \cdot V_{m}$

Comparing to steel which $(E / \rho) \approx 26$ it is seen, that for $\mathrm{TaC} /$ stellite composite higher ratio is available when the hardphase volume fraction is greater than $30 \%$. This is the result of high density of $\mathrm{TaC}$ ceramic which is almost 2 times the steel density. On the other hand, over 13\% hardphase volume fraction is needed to assess for the same specific density as steel in $\mathrm{TiC} /$ stellite composite.

\section{2}

Finite element analysis of macroscopic stress in the global structure

The finite element plane stress elastic analysis was conducted in order to obtain the stress and strain field in a global structure composed of a composite surface layer with $1 \mathrm{~mm}$ thickness and $10 \mathrm{~mm}$ thick low alloy steel. Fig. 8 shows the finite element mesh, which has 594 four node plain stress elements connected in 550 nodes.

The structure is assumed to be stress free and uniform temperature drop of $-1000 \mathrm{~K}$ is applied to simulate the formation of thermal residual stresses upon cooling from composite's fabrication (1293 K) to room temperature 

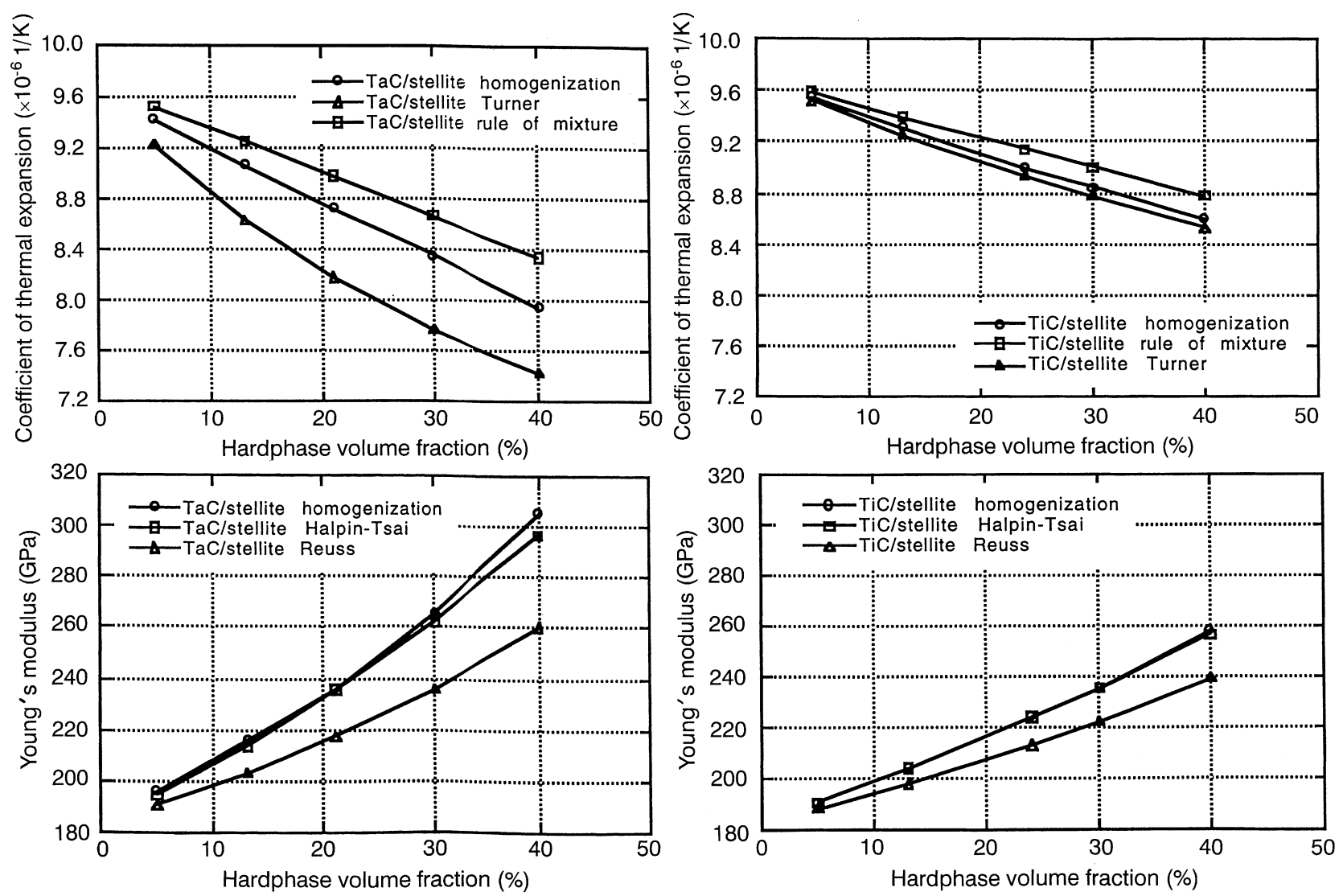

Fig. 6. Comparison of calculated homogenized properties of $\mathrm{TaC} /$ stellite and $\mathrm{TiC} /$ stellite composites with respect to hardphase volume fraction

(293 K). For the linear analysis any level of temperature loads could give the same quality results, however, the assumption of the real temperature drop would give us the view on the stress level comparing to the elastic limit of applied materials. It was also assumed in the calculations that there is a perfect contact on the boundary of the composite layer and substrate material. The anisotropic properties of composite are input in the analysis through the elastic and thermal expansion coefficients matrices and the properties of substrate material are assumed to be isotropic and a typical one is for steel: $\alpha=12 \cdot 10^{-6} 1 / \mathrm{K}, E=210 \mathrm{GPa}, v=0.3$. The material

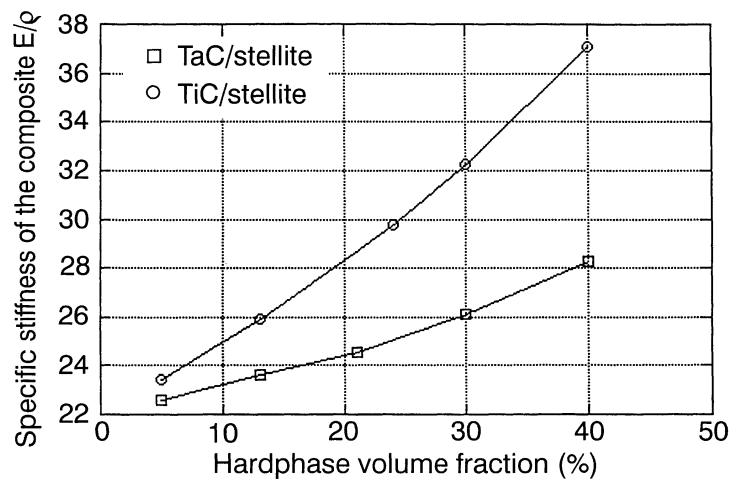

Fig. 7. Variation of composite specific stiffness with hardphase volume fraction for $\mathrm{TaC} /$ stellite and $\mathrm{TiC} /$ stellite composites properties are assumed to be constant in the analyzed range of temperature.

The finite element calculations show that the surface layer after cooling down to room temperature is in compression primarily by the influence of $\sigma_{x x}$ stress component parallel to the layer/substrate interface. The von Mises stress field shows the edges to be the regions of high stress concentration, but this singularity occurs in many systems composed of materials with different properties and can be a separate field of research in this area. For our purpose we will narrow our study to regions laying far

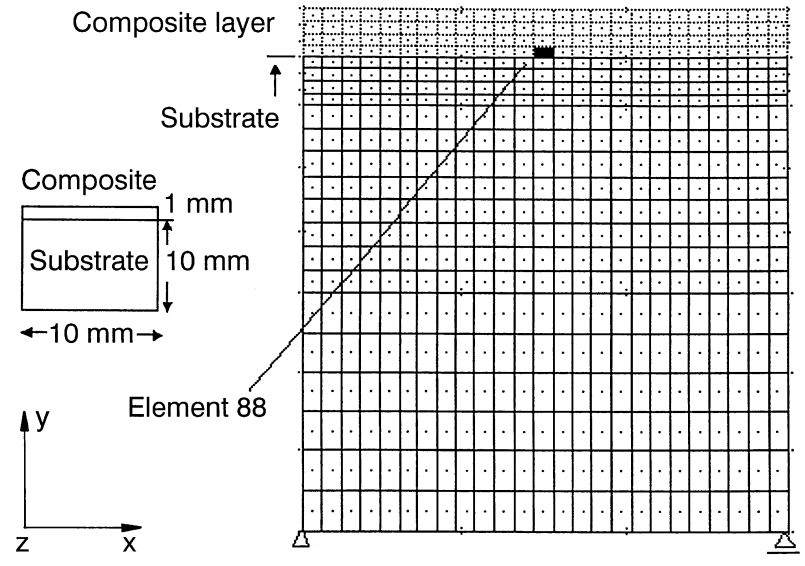

Fig. 8. The finite element model of the MMC surface layer produced over the low alloy steel 
from the edges. The $\sigma_{x x}$ stress and von Mises $\left(\sigma_{M}\right)$ stress distribution is presented in Fig. 9 for one of TaC/stellite models with $21 \%$ hardphase volume fraction.

The overall stress distribution for both composite systems and for each hardphase volume fraction is similar expect for the stress level. The stress level primarily depends on the difference of strains in the $x$ direction between the layer and the substrate resulting from different contraction of composite and substrate during cooling process of composite structure. This is because of the difference in thermal expansion coefficients between the composite and steel substrate. The increase of the hardphase volume fraction alters the homogenized composite's properties and as a result, the global stress level is also changed. As it was expected the residual stress in composite layer changes linearly with hardphase volume fraction because of the linearity of the problem. It is seen from the Fig. 10 that there is a symmetry across the zero stress line between $\sigma_{x x}$ and $\sigma_{M}$ stresses for both analyzed composites that confirms the main role of $\sigma_{x x}$ stress in the effective stress of the composite. It is also seen that for the small hardphase volume fractions there is a small differ-

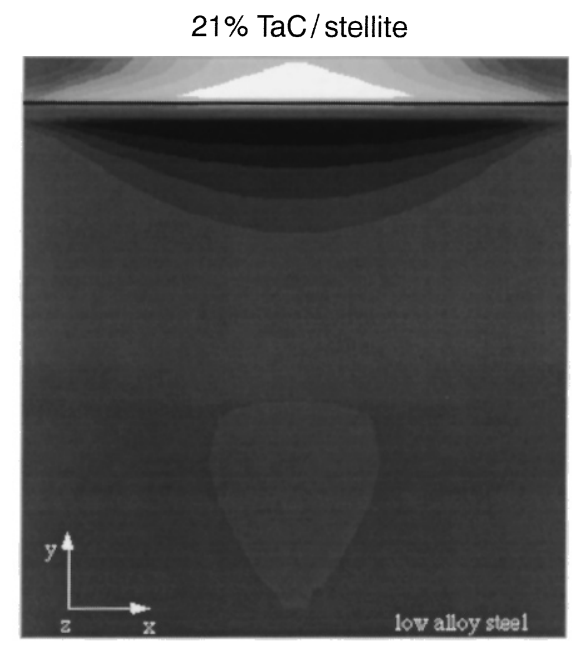

a

$21 \% \mathrm{TaC} /$ stellite
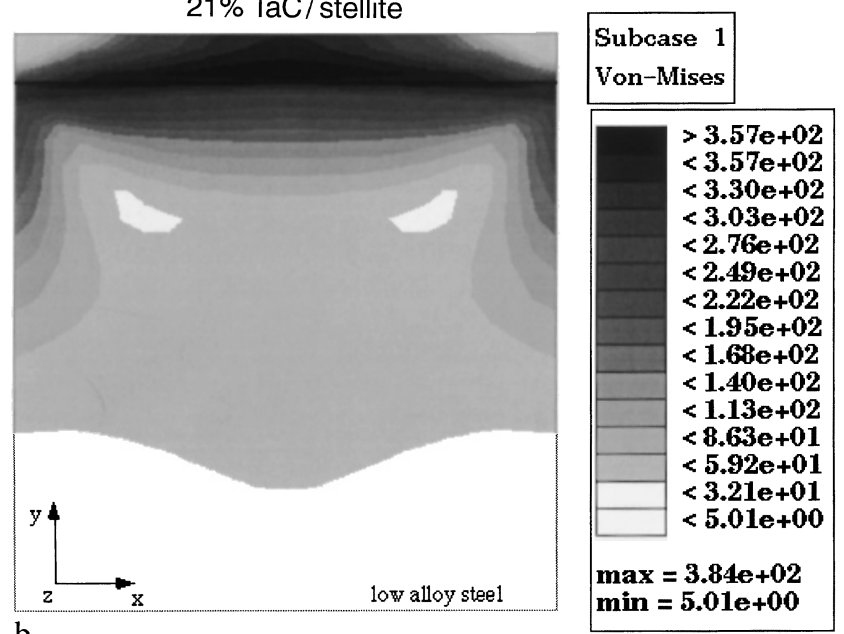

b

Fig. 9a,b. Distribution of $\sigma_{x x}$ macroscopic residual stress (a) and von Mises (b) residual stress calculated for $21 \% \mathrm{TaC} /$ stellite composite layer

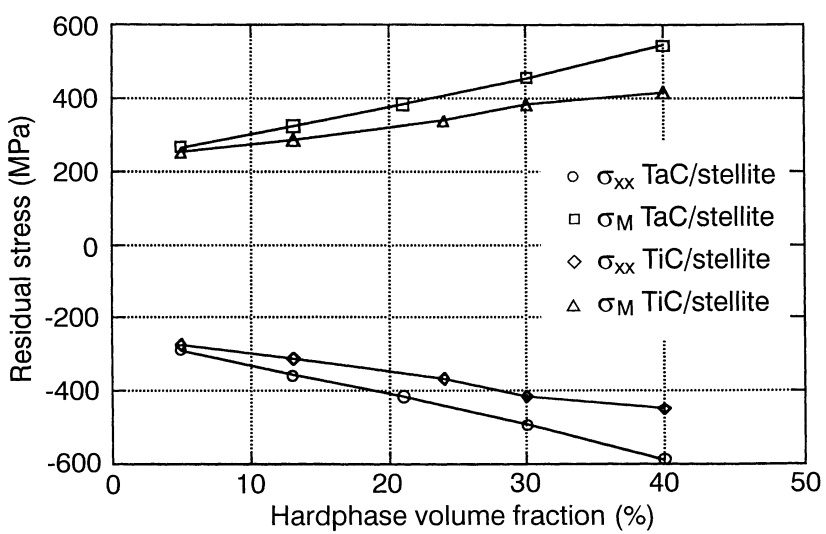

Fig. 10. Variation of macroscopic residual stress with hard phase volume fraction change for $21 \% \mathrm{TaC} /$ stellite and $24 \%$ TiC/stellite composites

ence in the stress level for $\mathrm{TaC} /$ stellite and $\mathrm{TiC} /$ stellite composites despite the differences in properties of $\mathrm{TaC}$ and $\mathrm{TiC}$ inclusions. Particularly, the ratio of $\mathrm{TaC}$ to $\mathrm{TiC}$ both $\sigma_{x x}$ and $\sigma_{M}$ stress equals $\approx 1$ for $5 \%$ inclusion volume fraction. This may result from a very close values of homogenized properties of both composites with $5 \%$ volume fraction, for which the effective composite properties depend mainly on the matrix properties.

The stress level in TaC/stellite composite is increased by about $100 \%$ from $5 \%$ hardphase volume fraction to $40 \%$, while for the TiC/stellite this increase reached about $64 \%$. Since the stress is compressive it is not seemed to be dangerous for the composite with hard ceramic inclusions. The variation of the von Mises stress and $\sigma_{x x}$ stress component from the surface through the thickness of the model shows the extreme of stress level just above the composite/substrate interface and the change for stress sign occurs in the substrate (Fig. 11). The $\sigma_{x x}$ stress in the substrate material may exceed the elastic limit near the boundary with composite layer, especially for the hardphase volume fraction greater than $20 \%$. This shall result in the redistribution of stress at the composite/substrate boundary and would influence the microscopic stress field.

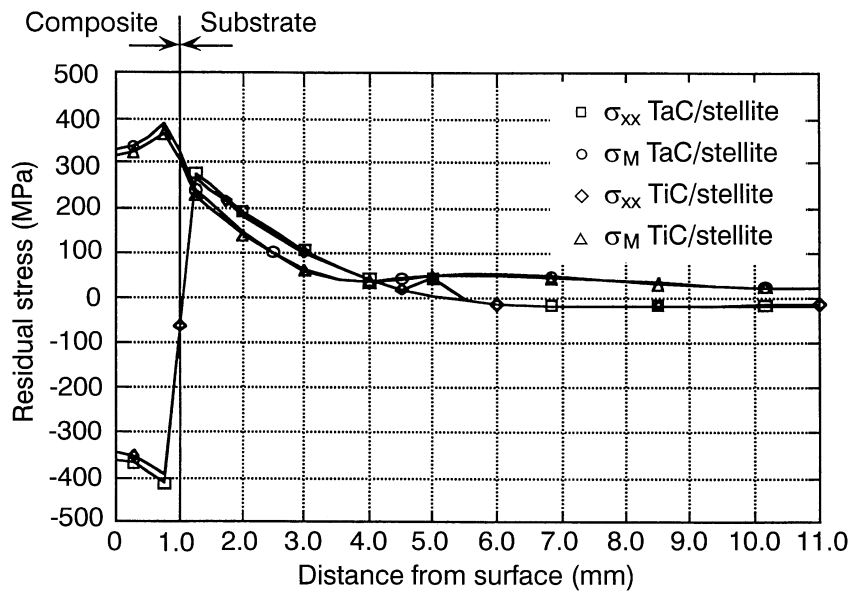

Fig. 11. Thermal residual stress profile across the surface of $21 \%$ $\mathrm{TaC} /$ stellite and $24 \% \mathrm{TiC} /$ stellite composite 


\section{3}

Microscopic stress representation in the unit cell

The results of the microscale thermal residual stress distribution are obtained for the composite unit cell taken from the boundary of composite/substrate interface (element 88 in Fig. 8), where the stress reaches the highest values according to the results of stress variation through the surface of the layer (see Fig. 11).

The local stress state in the micro scale is complex and depends on the hardphase volume fraction. Generally, ceramic inclusions contain most compressive residual stresses of magnitude few times higher than the macroscopic stress in composite layer. The matrix stays under weak tension in regions laying far from the inclusions or in compression close to the hardphase boundary as well as in regions laying between inclusions located close each other. There is no stress component which could dominate the overall composite stress response so the von Mises stress shall be the general indicator of the stress severity. Fig. 12 shows von Mises $\sigma_{M}$ thermal residual stress distribution within the unit cell for $21 \% \mathrm{TaC} /$ stellite and $24 \%$ $\mathrm{TiC} /$ stellite composites with a $3 \mathrm{D}$ plots of stress distribution.

It can be seen from these pictures that stress concentrates in regions laying between the particles and also near the inclusion/matrix boundary. In order to estimate the local stress in composite, von Mises stress was averaged and calculated separately for the hardphase and for the inclusion for the given unit cell. The variation of this averaged stress with hardphase volume fraction is presented in Fig. 13 for both analyzed composites. The increase of inclusion content results in considerable increase of the average stress in inclusions. The increase of the average $\sigma_{M}$ residual stress from $5 \%$ to $40 \%$ hardphase volume fraction is lower than for the global stresses, but the magnitude of the maximal $\sigma_{M}$ stress is 3 to 4 times the average stress in inclusion. Such localized high stresses can cause the early cracking of ceramic even the stress is compressive.

Also, high stresses may lead to the formation of a local plastic zone around the inclusions, which will result in the redistribution of this stress from the hardphase/inclusion boundary. Another reason for such a high stress concentration, especially at the high volume fractions, might be the shape of the inclusion boundary, which is not smooth because of randomly generated inclusion pixels in the process of volume fraction change. Thus, the torn shape of the boundary works as the additional source of geometric concentrator increasing the stress at the inclusion/matrix interface. The ceramic-metal interface is a
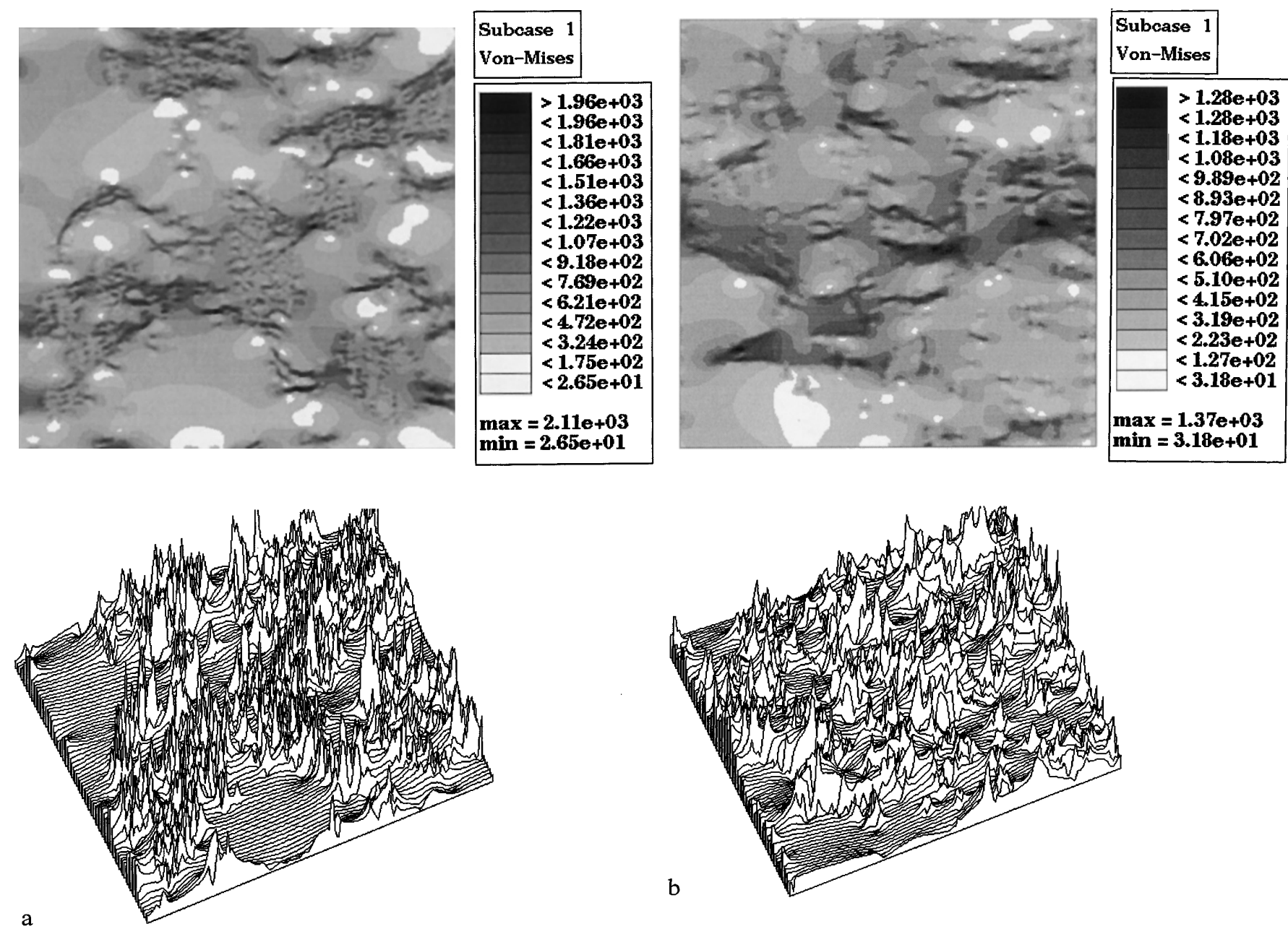

Fig. 12a,b. Distribution of local von Mises residual stress within the unit cell for $21 \% \mathrm{TaC} /$ stellite (a) and $24 \%$ TiC/stellite composites (b) 


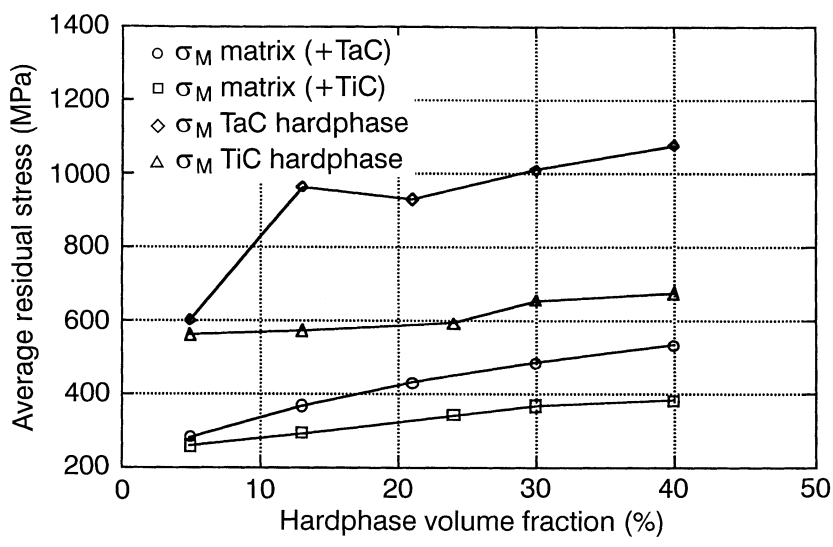

Fig. 13. Variation of the average microscopic von Mises residual stress within the unit cell for $21 \% \mathrm{TaC} /$ stellite and $24 \% \mathrm{TiC} /$ stellite composites

region where high stress concentration occurs. So better estimation of the stress severity would be obtained if the averaging scheme could be narrowed to the stress values taken exactly from the boundary.

\section{4}

\section{Selection between 2D and 3D model}

All previous results were obtained for 2D plane stress models. We have repeated the applied calculation scheme for a 3D model of $21 \% \mathrm{TaC} /$ stellite composite. The unit cell was extruded in the " $z$ " direction creating a 3D representation of $2 \mathrm{D}$ plane structure. The global structure is also extended in the " $z$ " direction to form a cubic substrate with $1 \mathrm{~mm}$ composite layer as shown in Fig. 14.

The homogenized properties calculated for the 3D model differ from those obtained in 2D analysis by 2 to $9 \%$ in the same $x-y$ plane. In out of $x-y$ plane the differences raised from 5 to $20 \%$. The results of stress distribution in 3D model, for $x-y$ plane are similar to those obtained in the $2 \mathrm{D}$ analysis except the stress level, which reaches more extreme values than for 2D case. From Fig. 15 presenting von Mises stress distribution we can see the extreme stresses exist also in the center of the model, which results from both $x x$ and $z z$ stress components as opposed to the edge planes, where one of the stress components reaches zero.

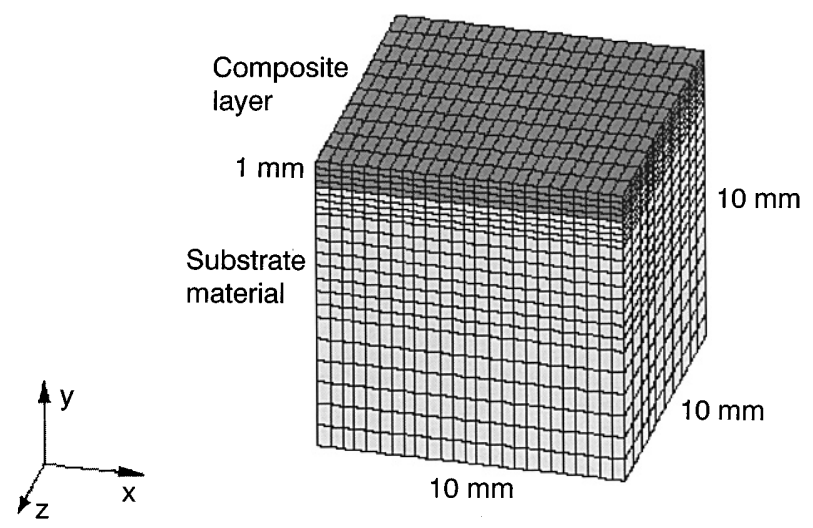

Fig. 14. 3D model of global composite structure for calculation of stress state.

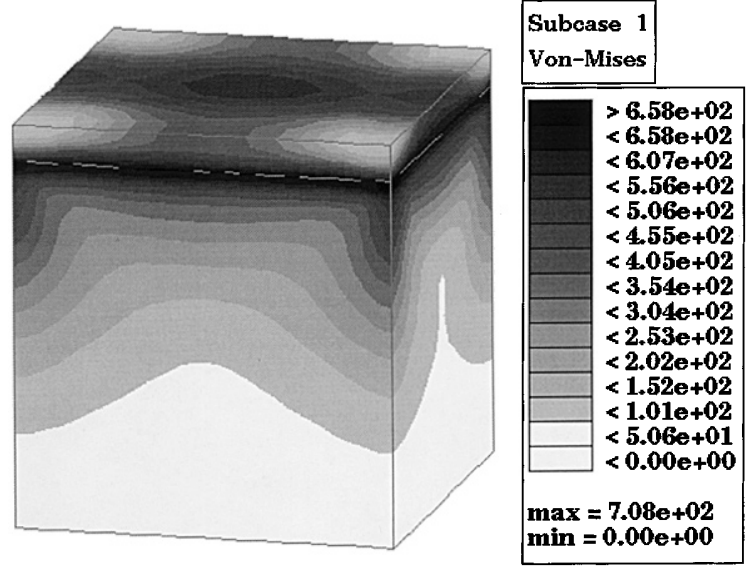

Fig. 15. von Mises global stress distribution for 3D 21\% TaC/stellite composite

The difference between these two models is also seen in the stress variation across the surface of the layer for the $x-y$ plane as shown in Fig. 16. The $\sigma_{M}$ stress profile tends to have sharp stress change at the composite/substrate boundary for the 3D model. The point of maximum $\sigma_{M}$ stress is shifted from the near-boundary to the boundary in comparison with the $2 \mathrm{D}$ case. The $\sigma_{x x}$ stress variation for $3 \mathrm{D}$ case is characterized by lower than $2 \mathrm{D}$ stress level in the composite layer with similar profile as for the $2 \mathrm{D}$ model.

The microscopic stress results obtained in $3 \mathrm{D}$ analysis shows similar to 2D model stress distribution in the unit cell, which is seen in Fig. 17 for $\sigma_{M}$ stress. The average local $\sigma_{M}$ stress is about 2 times higher in the hardphase and about 1.5 times the average $\sigma_{M}$ stress in the matrix for the $3 \mathrm{D}$ case anlysis.

The stress results calculated for 2D and 3D models show that a good agreement is obtained for the stress field both in macro and microscale. The selection between $2 \mathrm{D}$ or $3 \mathrm{D}$ analysis case should be carefully considered according to the complexity of the problem and computer efficiency. For a simple model analysis the $2 \mathrm{D}$ case could give sa-

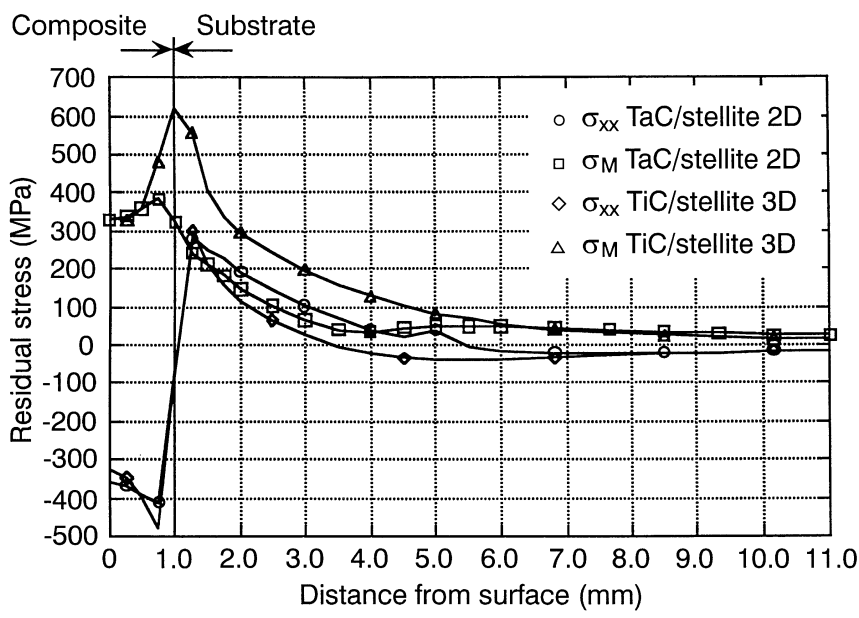

Fig. 16. Comparison of thermal residual stress profiles across the surface of $21 \% \mathrm{TaC} /$ stellite composite for $2 \mathrm{D}$ and $3 \mathrm{D}$ case stress analysis 

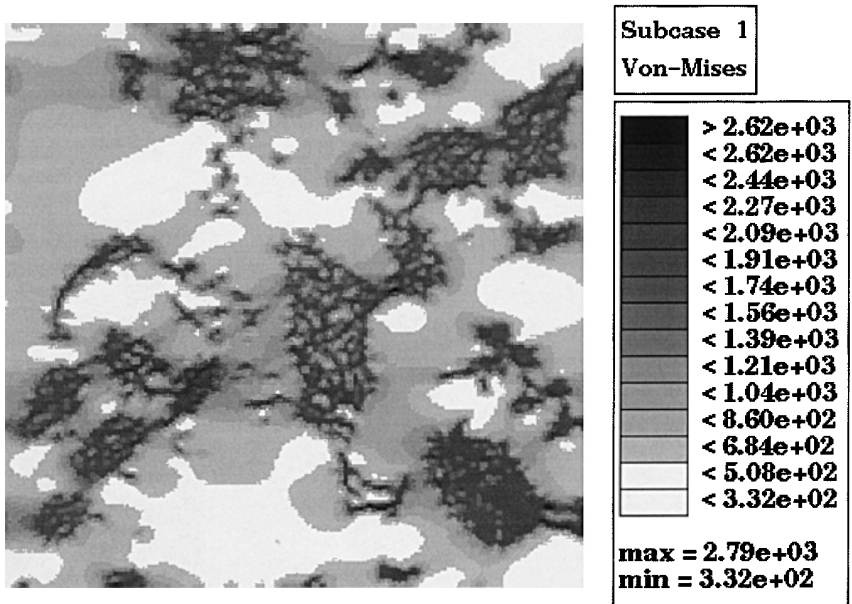

Fig. 17. Von Mises local stress distribution within the $x-y$ plane for 3D $21 \% \mathrm{TaC} /$ stellite composite

tisfactory results, especially for the purpose of comparison with other composite systems It is worthwhile to notice that the calculation time for $3 \mathrm{D}$ case is much longer than $2 \mathrm{D}$ one, as a result of the total node numbers, which for 2D case equaled about 30000 while for 3D case 90000 . This analysis has been conducted on HP 9000/715 workstation and the total computing time was 7 to 8 times longer for 3D model.

\section{5}

\section{Analysis extension for other hardphases}

The present method of homogenization combined with the digital-image processing and finite element calculations appears to be suitable to apply for other inclusion materials even though we do not have their microstructures. By using the microstructure of one composite material we can substitute its properties with other, so that different inclusions can be analyzed. This will give us comparable results of the thermomechanical behavior of composites with different hardphases. Such modeling may simplify the process of initial selection of inclusion material for a given matrix or a matrix for a given hardphase.

As an example we present the results of such analysis, which are based on the $\mathrm{TiC} /$ stellite composite microstructure with $24 \%$ of inclusion volume fraction. To analyze the effect of inclusion material we substituted $\mathrm{TiC}$ properties by $\mathrm{TaC}, \mathrm{SiC}$ and $\mathrm{HfC}$ ceramics assuming identical microstructure. Selected ceramics have different thermal expansion coefficients and different Young's modulus, so the combined effect of these properties on the stress level can be examined. We have studied the Young's modulus ratio and the CTE mismatch effect as they have the main influence on the stress state in MMC.

In the macroscopic stress analysis, the effect of the difference in the coefficients of thermal expansion (CTE) between the steel substrate and composite layer, and the effect of the ratio of composite Young's modulus $E_{h}$ to the Young's modulus of steel substrate $E_{s u b}$ are presented in Fig. 18a and Fig. 18b respectively. It is seen form these pictures that the high Young's modulus of $\mathrm{TaC}$ inclusion contributes to the elevated stress level in the composite.
We may assume that the smallest ratio of $E_{h} / E_{\text {sub }}$ will not guarantee the lowest stresses, which is clearly seen for the $\mathrm{SiC} /$ stellite composite having the lowest CTE of SiC ceramic among analyzed inclusions.

Therefore, the CTE mismatch between the composite and substrate will play the main role in the generation of stresses, although the high Young's modulus of $\mathrm{TaC}$ inclusion is responsible for higher stress level comparing to $\mathrm{HfC}$ and TiC inclusions which have higher CTE mismatch. The influence of high Young's modulus of inclusion on the stress increase is seen especially at higher volume fractions where the effect of inclusion properties on the effective properties of composite become evident which is opposed to the low volume fractions. Similar trends are observed for the average microscopic stress within the unit cell as seen in Fig. 19, where the von Mises stress is plotted separately for the matrix and inclusions. Herein, the CTE mismatch refers to the difference between the matrix and the hardphase while the Young's modulus ratio to the hardphase and matrix.

The results of the CTE mismatch show clearly that the Young's modulus of $\mathrm{TaC}$ hardphase ceramic substantially increased the stress level in the hardphase, although the influence of the CTE mismatch seems to affect the stress level in a higher degree, which is shown for $\mathrm{SiC}$ inclusion. In both scales of stress analysis the CTE mismatch has the
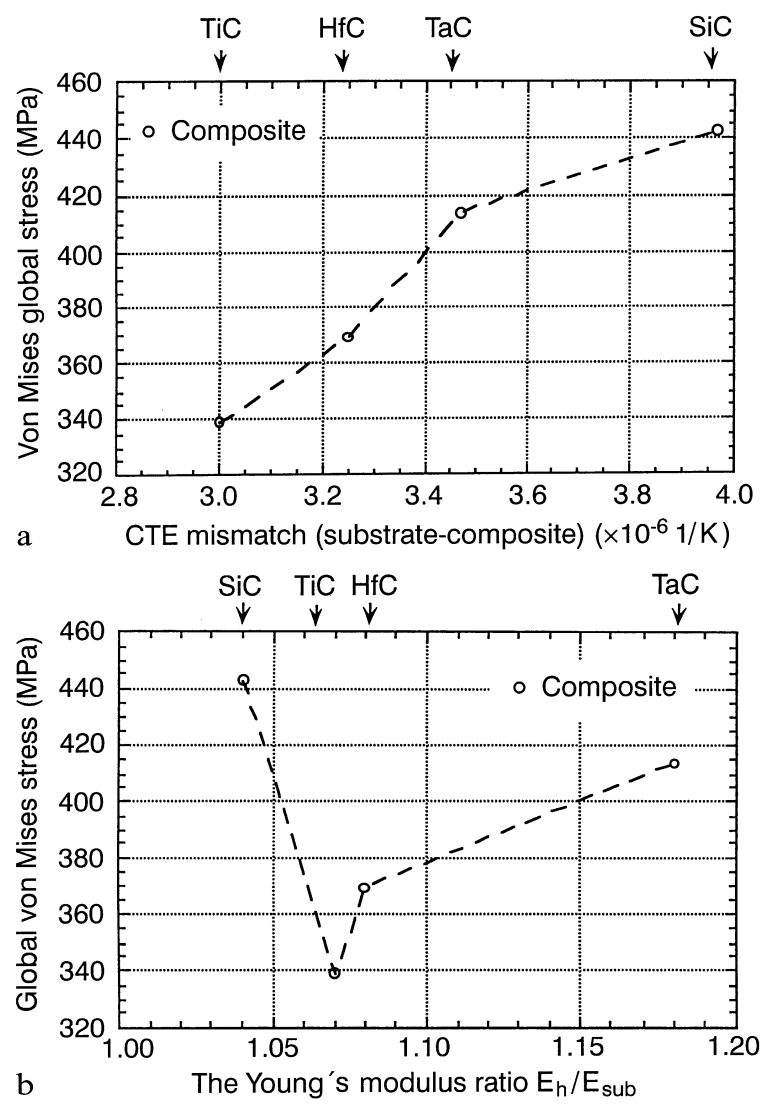

Fig. 18a,b. Influence of thermal expansion coefficient mismatch between the substrate and composite layer (a) and Young modulus ratio of the composite to the substrate (b) on the von Mises stress in the composite layer calculated for $24 \% \mathrm{TiC}, \mathrm{HfC}, \mathrm{TaC}$ and $\mathrm{SiC} /$ stellite composites 

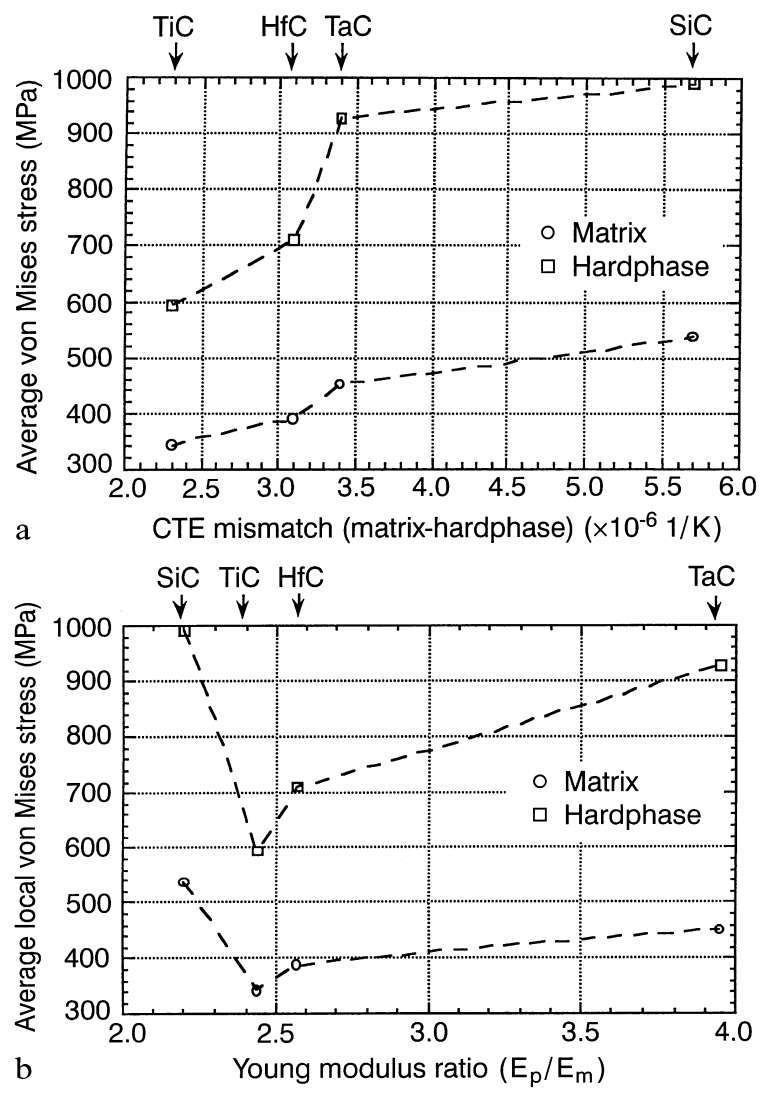

Fig. 19a,b. Influence of thermal expansion coefficient mismatch between the matrix and inclusion (a) and Young modulus ratio of the inclusion to the matrix (b) on the average von Mises stress within the unit cell calculated for $24 \% \mathrm{TiC}, \mathrm{HfC}, \mathrm{TaC}$ and $\mathrm{SiC} /$ stellite composites

primarily effect on the stress level in the analyzed composites. The Young's modulus effect becomes visible for high ratios of $E_{p} / E_{m}$. This is seen in Fig. 19a for the HfC and $\mathrm{TaC} /$ stellite systems which have similar CTE mismatch, but the stress level sharply increased in $\mathrm{TaC} /$ stellite composite because of high Young's modulus of $\mathrm{TaC}$ ceramic. In the above analysis we assumed no influence of temperature on the CTE and Young's modulus $(E)$ of composite material. The CTE of most ceramics and metal increases with temperature while the $E$ decreases. Therefore, some authors use the product of $(C T E * E)$ as a constant value over the analyzed range of temperature and treat it as a measure of joint effect of both the CTE and $E$ on the stress level in a structure. Such relation is presented for studied composites in Fig. 20 and shows that the $\sigma_{M}$ stress reaches minimum for CTE $* E \approx 2$. Although the CTE and $E$ effect on the stress level is not equal we may use this simple relation in the evaluation of stress for many composite systems.

\section{6}

\section{Summary}

We have presented some aspects on modeling macro and microscopic thermal residual stresses in the metal matrix composites by the homogenization method with application of digital image-base technique. The process of deposition advanced composite materials onto the substrate material by using plasma spraying combined with electron

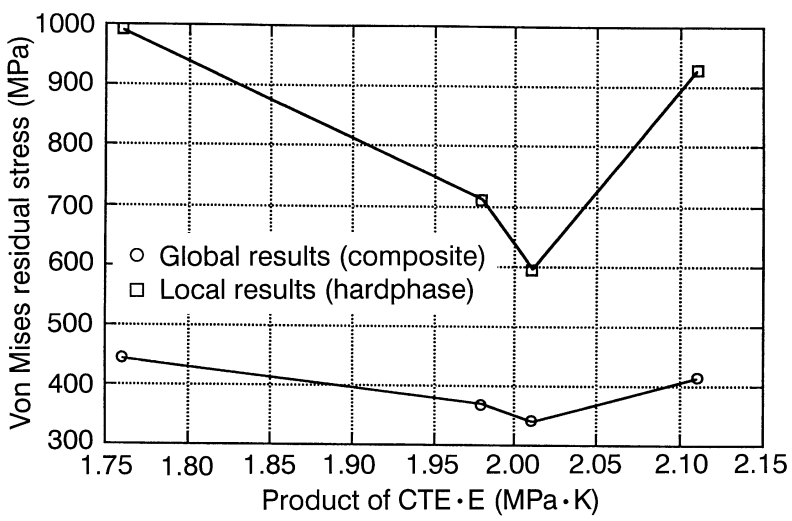

Fig. 20. The relation between the product of homogenized properties CTE*E of stellite-based composite reinforced with different ceramic inclusions and von Mises stress calculated for global and local scale

beam remelting method is very attractive for producing surface layers with enhanced strength and resistance to external loads comparing to traditionally used materials. The embeding of ceramic inclusions in the metal matrix inevitably leads to the generation of internal stresses during the formation and in service life of composite structures. Upon the scale of mismatch in thermophysical properties of composite's constituents, the high stress may reduce substantially the service life of the structure. In the extreme case the composite may be damaged by crack propagation through the matrix or inclusions. Therefore the stress estimation in the MMC structures becomes important.

In the present work the homogenization method was limited to the linear elastic range. The application of elastic-plastic analysis for that kind of microstructures could be difficult to conduct nowadays as it requires much larger computer memory and calculation times. The assumption of linear elasticity is valid only for hard inclusions like advanced ceramics which we may treat as elastic bodies. The stress field in the global structure was calculated using homogenized properties $(E, G, V, \alpha)$ of studied composites. It is shown that these properties strongly depend on the hardphase volume fraction. The results of stress level indicate that the plastic field may be built up in a thin layer of substrate material just close to the boundary with composite layer. As a result the global stress level in the composite layer may change, which will affect the microscopic stress within the composite's unit cell.

Upon the results of microscopic stress distribution in the unit cell of studied composite systems, we may say that the residual stress field is complex while inclusions are generally under compression. There is a high stress concentration at regions close to the inclusion/matrix boundary. The magnitude of the stress in the stellite matrix is much above the matrix elastic limit, which suggests that a local plastic field may be developed around ceramic inclusions and as a consequence the stresses shall be redistributed from the region of stress concentration.

The change of hardphase volume fraction is a very attractive feature of the analysis. Without the need to produce composite structures with specified hardphase volume fractions we can simulate the thermomechanical 
response of composite materials in the process of composite design. This is especially important for simulation of stresses in composite layers which are produced by direct deposition onto the substrate material, because of the difficulty to control the real hardphase volume fraction. The calculations we have conducted for TaC/stellite and $\mathrm{TiC} /$ stellite composites with hardphase volume fraction ranging from 5 to $40 \%$ reveals that the thermal residual stresses both in the macroscale (global structure) and in the microscale (unit cell) of different composite systems, do not vary substantially for the low (5\%) hardphase volume fractions. With the further increase of hardphase content in the matrix the difference is expanding to reach the maximum at the highest volume fractions of inclusions.

In the analysis of thermal residual stresses the main role play the properties of constituent materials. The most important is the mismatch in thermal expansion coefficients between the hardphase and the matrix in the microscopic stress analysis and between the composite layer and the substrate material in the macroscopic stress analysis. Also, the stiffness of the local and global structures characterized by Young's modulus ratios affects the stress generation in the ceramic/stellite composite systems. Therefore, the selection of the hardphase material for a given matrix is of essential meaning. We have shown the influence of hardphase material on the stress level for by selecting $\mathrm{TaC}, \mathrm{TiC}, \mathrm{SiC}$ and $\mathrm{HfC}$ ceramic inclusions with $24 \%$ volume fraction in the stellite matrix and assuming identical composite microstructure. It is evident that the increase of mismatch in the thermal expansion coefficient between the matrix and the inclusion as well as the increase of inclusion stiffness leads to the higher stresses in the composite, although the CTE mismatch seems to dominate the stress generation. On the other hand, if we consider the composite specific stiffness, then we will see that $\mathrm{HfC}$ and $\mathrm{TaC}$ ceramics have very high density, which can make them less attractive than conventional materials.

The selection between 2D and 3D models for the stress analysis requires consideration of the efficiency of computer calculations versus obtained results. We have shown that the 2D model applied in the stress analysis gives comparable results with the 3D one allowing for the substantial reduction of computing time and memory. Therefore, the application of 3D models shall be carefully considered according to the analysis we are going to conduct and expected results.

It is important to remember that the stress analysis presented in this paper refers to the thermal residual stresses generated during the composite fabrication. In the service life of the composite structure other stress fields may develop resulting from the external service loads. These stresses will superimpose with the residual stresses, which can lead to a new, different stress state. If we expect the composite structure to work under tensile loads then the compressive residual stresses are preferred in the composite as the overall stress level will be reduced. Although tensile stresses are generally more dangerous for the brittle ceramic materials, the high compressive stresses within the composite layer are not preferred too, because they can results cracking or delamination a thin composite layer from the substrate.

The method presented in this paper can be an adequate tool to compare the macro (global) and microscopic (local) stress state in the metal matrix composites with hard inclusions, as well as to obtain the homogenized composite properties. We shall emphasize, that this kind of analysis is unique as it allows the real microstructures of composite materials to be analyzed. The presented calculation scheme may be applied to other kinds of loading or the combined effect of multiple loads can be analyzed.

Further development of this method would allow to account for the plastic deformation in the elastic-plastic analysis. At the present stage of computer, it is still a difficult task to deal with realistic complex microstructures.

\section{References}

ASM Engineering Materials Reference Book. ASM International Metals Park, OHIO 44073: 150-188

Brountman, L. J.; Krock, R. H. (1974): Metallic Matrix Composites. In: Kreider K. G. (ed): Composite Materials, vol. 4, Academic Press, New York

Chawla, K. K. (1987): Composite materials. Science and Technology. Springer Verlag

Cheng, C.-H. (1992): Modeling of the thermal elasto-plastic behaviour for composite materials using homogenization method. Ph.D. thesis, The University of Michigan

Devries, F.; Léne, F. (1987): Homogenization at set macroscopic stress: Numerical Implementation and Application. Recherche Aerospatiale 1: $34-51$

Duvaut, G.; Nuc, M. (1983): A new method of analysis of composite structure, Ninth European Rotor Craft Forum, Paper No. 88, Stresa, Italie

Guedes, J. M.; Kikuchi, N. (1990): Preprocessing and postprocessing for materials based on the homogenization method with adaptive

finite element methods. Comp. Meth. in Appl. Mech. and Engin. 83: 143-198

Halpin, J. C.; Kardos, J. L. (1967): Environmental factors estimation in composite materials design. AFML TR: 67-423

Hollister, S. J.; Kikuchi, N. (1994): Homogenization theory and digital imaging: a basis for studying the mechanics and design principles of bone tissue. Biotechnology and Bioengineering 43 (7): 586-596

MacKay, R. A. (1990): Effect of fiber spacing on interfacial damage in metal matrix composites. Scripta Met. 24: 167

Kerner, E. H. (1956): Proc. Phys. Soc., London, 69B: 808-813

Matejka, D. (1989): Plasma spraying of metallic and ceramic materials. New York, J. Wiley

Morrell, R. (1989): Handbook of properties of technical and engineering ceramics. Part I. National Physical Laboratory, London, HSMO

Properties of Deloro Stellite Alloys (1970): Deloro Stellite Ltd. B43/C Reuss, A. Z. (1929): Berechnung der Fliessgrenze von Mischkristallen auf Grund der Plastizitätsbedingung für Einkristalle. Angew. Math.

Mech. 9: 49

Rohatgi, P. K.; Ray, S.; Lin, Y.; Narendranath, C. S. (1994): Tribological properties of composites containing hard and soft particles. In: Rohatgi P. K. (ed), Friction and Wear of Technology for Advanced Composite Materials. ASM International 1-19

Shi, N.; Arsenault, R. J. (1994): Plastic flow in SiC/Al compositesStrengthening and ductility. Annu. Rev. Mater. Sci. 24: 321-357

Taya, M.; Arsenault, R. J. (1989): Metal matrix composites. Thermomechanical Behaviour. Pergamon Press

Terada, K.; Kikuchi, N.; Miura T. (1996): Digital image-based modeling applied to the homogenization analysis of composite materials, submitted to computational mechanics.

Turner, P. S. (1946): J. Res., NBS 37: 239 
Sanchez-Palencia, E. (1980): Non-homogeneous media and vibration theory, Lecture Notes in Physics 127, Springer-Verlag, Berlin

Vaidya, R. U.; Chawla, K. K. (1994): Thermal expansion of metal

matrix composites. Composites Science and Technology 50 (1): 13-22
Whitney, J. M.; McCullough, R. L. (1990): Micromechanical materials modeling, In: Gillespie J. W. and Carlsson L. A. (ed): Delaware Composites Design Encyclopedia, Technomic Publishing Co. Inc. Lancaster, vol. 2: 99 